\section{Information Infrastructure for an Integrated Healthcare Services Network}

\author{
Manolis Tsiknakis ${ }^{1}$, Dimitrios G. Katehakis ${ }^{1}$, \\ Stelios C. Orphanoudakis ${ }^{1,2}$ \\ ${ }^{1}$ Center for Medical Informatics and \\ Health Telematics Applications (CMI-HTA), \\ Institute of Computer Science, \\ Foundation for Research and Technology - Hellas \\ email: \{tsiknaki , katehaki, orphanou\}@ics.forth.gr, \\ ${ }^{2}$ Department of Computer Science, University of Crete.
}

\begin{abstract}
The growing demand for more efficient and effective healthcare services, coupled with an implicit requirement for supporting citizen mobility and continuity of care, is currently setting the stage for the exploitation of Information and Communications Technologies, also known as Information Society Technologies, in the healthcare sector.

The current vision comprises affordable access to healthcare resources and services for all citizens, thus making medical expertise a shared resource wherever and whenever needed. Important areas in which Information Society Technologies are likely to have a significant impact include those of pre-hospital health emergencies, remote monitoring of patients with chronic conditions, and medical collaboration through sharing of health-related information resources. Accessibility to these and other media-rich, user-oriented services, in the context of the emerging Global Information Society, will be supported by a Healthcare Information Infrastructure (HII), which can achieve effective horizontal integration of networked information sources.
\end{abstract}

Keywords: integrated healthcare network, healthtelematics services, healthcare information infrastructure, and electronic health record

\section{Introduction}

The current trend in healthcare information technology is increasingly digital and multimedia-oriented. The next generation of healthcare information systems will consist of a large number of heterogeneous, autonomous, and distributed information systems, knowledge-intensive applications, and large quantities of multimedia medical data. Hence a key challenge facing researchers and system developers is to provide a new organizational framework that can integrate this heterogeneous collection of resources into what appears to be a uniform conglomeration of data and knowledge to increase the availability of previously inaccessible information and to address the demanding information processing requirements of modern medical applications [1].

The creation of the Healthcare Information Infrastructure (HII) is driven by, among other factors, the need for:

- data on outcomes of medical cases that will enable effective choices and compensation of providers,

- automation of mundane tasks to place the focus on patient needs rather than paper-work,

- empowerment of consumers to become more actively involved in their own healthcare,

- flexible remote access to relevant information in order to ensure continuity of care,

- continuous process improvement through integrated and distributed information technology.

Existing and emerging Information Society technologies provide technological solutions to the complex problems involved in any effort at developing the healthcare information infrastructure.

Our center is currently involved in an effort to design and implement a reference architecture, and to deploy the necessary HII and applications, for the provision of integrated services in HYGEIAnet, the healthcare network of Crete. This paper reports on the current status of this ongoing effort. 


\section{Current Trends}

Priorities for the 21st century are linked to the shift towards citizen-centered services, meeting Quality of Service (QoS) requirements, and a substantially greater emphasis on wellness and prevention. Healthcare professionals will continue to deliver care, but will also be increasingly required to share their knowledge and expertise with other colleagues and citizens in general.

The new paradigm provides that informed citizen care for their own health and, together with other cooperating stakeholders, who may also be responsible for the continuity of health services within a region, have an impact on the operation of the healthcare system as a whole. In this context, decentralized healthcare can be supported by integrated services for seamless and personalized information delivery, while services and information must be accessible without visible organizational boundaries.

The Health Telematics Services that are being developed and are partially in use address the needs of all involved actors. Potential users of such services are (a) health administrators, (b) regional and national health authorities, (c) healthcare professionals, and (d) patients and citizens at large. Specifically,

- for health administrators and the regional and national health authorities, continuity of care results in a reduced number of repeated exams, better monitoring and management of healthcare procedures, and better use of available, clinical, technical and financial resources.

- for healthcare professionals, the secure exchange of medical information for teleconsultation purposes, the requirement for efficient access to a patient's distributed Electronic Health Record (EHR), and the increasing demand for improved quality of services and evidencebased clinical practices create a need for the implementation of increasingly sophisticated information access and management tools.

- for patients and all citizens, one must provide access to information and services anywhere and anytime, while supporting mobility and continuity of care.

\section{Application Domain}

The healthcare domain is characterized by a hierarchical structure and involves a large number of specialties and user groups, each with different skills and requirements. The geographical distribution of resources is non-uniform and in many cases these are located in remote and isolated areas. The large volume of available information is inherently heterogeneous and multimedia in nature, while new information is produced at a very high rate.

Health Telematics can be used to provide a broad spectrum of integrated services in the areas of prehospital health emergency medicine, primary care, hospital care, and home care. The traditional environment of autonomous clinical information systems, that provide point-to-point connections so that telemedicine experiments can be conducted, contributes to the fragmentation of information that cannot be used efficiently to utilize the potential of the underlying technologies. Integrated regional healthtelematics networks (on top of Figure 1) are currently at advanced stages of development and aim to provide round the clock services to the citizen, not limited by certain resource availability constraints.

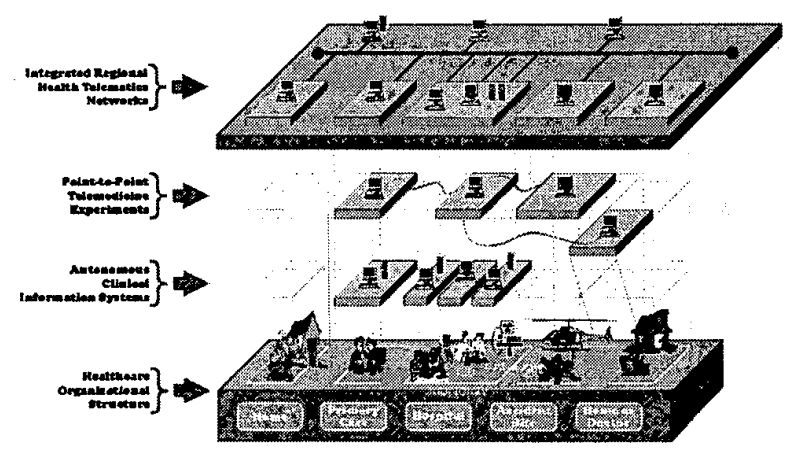

Figure 1: Evolution of the healthcare environment.

A fundamental prerequisite for the establishment of a scaleable regional health telematics services network is the development of the architecture and tools for 
the integration of specialized autonomous applications that are supported by the HII [2]. The effective horizontal integration of networked information services can provide support for the interoperability of applications and services within a healthcare institution, and the intelligent management of health related information within such an integrated network.

\section{Health Telematics}

Health telematics involves the use of information processing tools and telecommunications to provide the functional and operational framework that allows computers to transfer medical data to one another. Interoperability among telematic services and standalone applications is critical, since services and applications share data and information. Hence, telematic services need to be part of a collaboration environment that ensures the continuity of care and information sharing, under strict security and authorisation policies. Health telematics services, typically required within a regional healthcare network, may be classified into five basic classes: collaboration, resource, educational, integrated electronic health record (I-EHR), and added-value services.

Collaboration services bridge the gap created by the physical distance separating the various users, while promoting social interaction and the exchange of vital information. This type of user-oriented services includes tele-consultation and tele-monitoring. Teleconsultation sessions among healthcare professionals may compensate for the lack of experienced or specialized personnel at remote sites, at the site of an accident, or at primary care facilities. Telemonitoring services establish patient-physician or physician-physician collaboration for the purpose of delivering healthcare at the home of the patient or monitoring a medical examination remotely.

Resource services provide information on the availability of physical resources such as hospital departments, diagnostic modalities, mobile emergency units and their characteristics, through regional white and yellow page directories. In recent years, the Internetbased web technology has stimulated the creation of many such yellow pages by hospitals, health authorities etc., making it possible to get practical information about healthcare providers.

Educational services are especially important for rural regions, where access to information sources is limited. In order to raise public awareness, and to effectively support continuity of care, healthcare professionals must have access to educational material contained in digital medical libraries. Similarly, the public should have access to information relevant to diseases common in their region. Geographic isolation may not hinder the sharing of knowledge among professionals, who may improve the quality of service they provide through interaction with repositories of medical cases and other educational material.

I-EHR services support single-point access to clinical information by both patients and physicians. They provide a uniform view of data, which may be configured differently at different locations. This is necessary due to the fact that segments of a patient's electronic health record may reside at different healthcare facilities visited by the patient.

Added-value services extend all the above services and provide specialized support to healthcare professionals. Image processing services, access to specialized indexing and search engines, information filtering, content-based image indexing and retrieval, and decision support are examples of services that facilitate efficient utilization of the information space.

\section{Architectural Framework}

Interoperability and the use of open standards are fundamental requirements in any effort at developing an architectural framework for the provision of health-telematics services. The implementation of a scalable, extensible and modular architecture further requires that the installed HII can continue to evolve and that, as technologies mature, new and improved capabilities can be taken advantage of.

The development of HYGEIAnet on Crete is targeted at providing an integrated environment for healthcare delivery and medical training across the island. 
HYGEIAnet takes advantage of the increasing capacity of terrestrial and mobile communication networks and the development of advanced telemedicine services to provide every citizen of the island with effective healthcare services and to support remote consultation among healthcare professionals in specialised centres, district and regional hospitals, and other points of care.

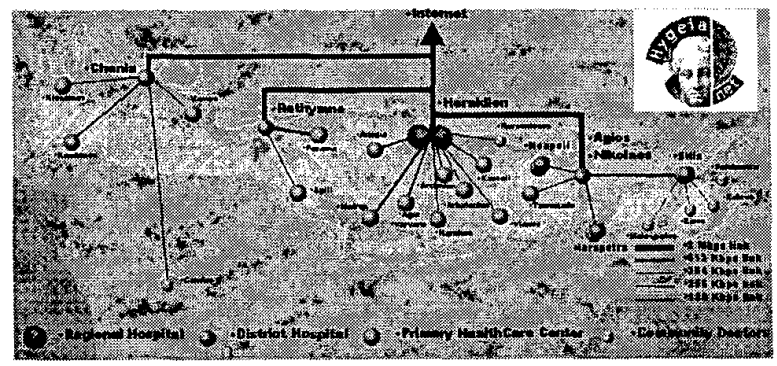

Figure 2- HYGEIAnet, the regional health telematic services network of Crete.

In the course of designing and implementing HYGEIAnet, special efforts are being made to meet the requirements of the user groups involved and to use state-of-the-art technology and standards at every stage. Alternative patient, problem, and case-based architectures for the I-EHR have being considered in an attempt to provide transparent access and secure communication of information between medical speciality areas, as well as in a variety of situations from community to hospital care across the region.

Various strategies for multi-database integration and interoperability were considered, in conjunction with the EHR, to provide homogeneous access to its distributed segments at all levels of the health care system. Advanced multimedia telematics applications supporting synchronous and asynchronous teleconsultation and co-operative diagnostic work between health care providers at different locations have also been developed and are part of the integrated system.

\section{Components of the HII in HYGEIAnet}

The application layer of the reference architecture consists of applications, which support user activities in the various areas of an organization. These applications are both information sources and/or information access points. Clinical, diagnostic, and administrative information systems, diagnostic imaging repositories, medical libraries, and user-oriented services are all part of the application layer. All applications and services of the application layer make use of their own data model and user-interface.

The core infrastructure components of HYGEIAnet support the secure access to hierarchically structured information on end-user personalized preferences, as well as management of other resource information, by means of a Healthcare Resource Directory (HRD) and the Patient Clinical Data Directory (PCDD). The latter is a central element of the HII. Additionally, the WebOnColl collaboration infrastructure allows for the secure creation and management of virtual workspaces, and telemedicine session folders.

The PCDD is a central middleware element of the overall architecture that provides information on the distributed EHR segments maintained by autonomous information systems [3]. Access to detailed information regarding specific healthcare encounters is provided subject to role-based authorization privileges and controls. The administration of the healthcare organizations' business rules, for different usergroups, is made via a specially tailored rule-editor [4]. In its current version the PCDD offers access to the clinical information systems (the legacy feeder systems) of different healthcare facilities in the region of Crete.

The collaboration infrastructure of WebOnCOLL is based on virtual workspaces and user profiles [5]. Virtual workspaces implement collections of heterogeneous objects, maintain history data, and support awareness regarding content updates and user interaction. Agents (human or software) connected to a workspace may add objects to the workspace and are notified of changes in the contents of the workspace, or when other agents are connected to the same workspace. User profiles enable the customization of workspaces according to user authorities, tasks, and preferences. The interoperability of WebOnCOLL 
with enabling middleware services of the regional HII ensures availability of accurate resource information and certification of the exchanged data.

Authentication services allow access only to authorized end-users. They are implemented by means of a security server that manages the roles and corresponding access rights of users, together with associations to personalized profiles. Its purpose is to certify the role and authority of both users and services (or applications) within the regional healthcare network. In this context, any patient is able to have complete access to all personal information.

Encryption services are responsible for the secure communication of sensitive personal information over the Virtual Private Network (VPN) of HYGEIAnet, as well as over the Internet. The combination of digital signatures for authentication, public key cryptography for recipient authentication, and Secure Socket Layer (SSL) protocols for secure datatransfer, provide the necessary technological framework for secure communication of healthcare related information across the Internet.

Patient identification services allow for the unique association of distributed record segments to a master patient index. This is a challenging task to perform and one of the major barriers towards developing a reliable I-EHR environment. This is because no global, unique person identification exists worldwide today. In addition, even for countries that posses one, this is not used internally for identification purposes by clinical or administrative information systems.

Auditing services are responsible for recording all interactions occurring between middleware services and/or end-user applications. The logs produced can then be used for both tracing back transactions in time, or even for charging. It is also used as a profiler tool for individual server loads, and for the construction of end-user interest profiles through data mining.

Resource location services are used for identifying available healthcare or computer resources and the means for accessing them. Examples of resources include pharmacies on-duty, hospitals and clinics, clinical information systems available at a regional level, methods and technologies available for accessing primary information, and protocols for exchanging information with them.

Encounter location services are used for locating primary information found in different patient record segments. In this context, encounter is the term used for describing clinical information that is produced during the communication about the patient, between two or more individuals, at least one of whom is a member of the responsible healthcare team.

Clinical Observation Access Services are required for obtaining primary information directly from specific clinical information systems. This service requires the implementation of standardized gateways for each clinical information system for securely importing/exporting patient record data. Certain filters are supported for the better location of information based on time and examination type criteria.

User Profile Services track the long-term interests of users and are used for maintaining personalized preferences. User profiles are initially created according to existing stereotypes that are automatically assigned to them, after gathering some initial userspecific information. Users may also actively contribute to the incremental building of their profiles.

Terminology Services have a dual objective: to map and associate existing coding schemes, and to relate the internal semantics of the clinical information systems maintaining primary information. The first objective is accomplished by means of UMLS, while the second requires a conscious effort by clinical information system developers and people responsible for maintaining I-HER services up to date.

\section{The integrated Electronic Health Record}

Based on a number of the components and services of HYGELAnet's infrastructure an I-EHR environment has been developed. Its objective is to deliver an encounter-centered view of the patient's EHR. It utilizes standardized CORBA interfaces to provide a consistent way to locate, access and transmit secure 
information about a patient's EHR segments. The IEHR is a front-end to an EHR indexing service, managed by PCDD, which indexes both structured and unstructured information that is provided by cooperating information systems. Apart from the lifeline view of all the available encounters of the patient, two more views are currently supported: a perclinical system view of encounter's history, as well as the traditional tabular view of old generation GUIs. Certain filters are supported for the better isolation and location of information based on time and examination type criteria.

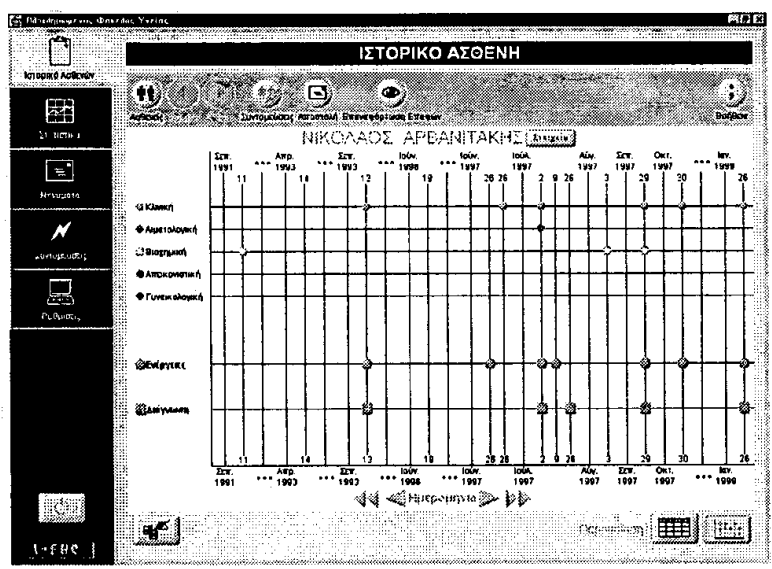

Figure 4: Human Computer Interaction with the I-EHR environment.

\section{Conclusions}

This paper presented the architecture and primary components of the health information infrastructure of HYGEIAnet, the integrated regional health telematics network of Crete. In its current state of development, HYGEIAnet provides a concrete example of an open, modular, extensible infrastructure based on related standards, for the next generation regional networks.

HYGEIAnet builds on a regional HII to improve the quality and accessibility of healthcare services and to enable their remote delivery. The open issues that remain to be resolved are many, but experience has shown that any carefully planned component-based architecture can support a wide range of advanced health telematics services. High priority issues for the near future are (a) the implementation of semantic mapping and terminology services so that information can be represented in a semantically unified way, (b) the establishment of a more flexible security infrastructure, and (c) data mining and knowledge extraction services to fully exploit the available information.

\section{Acknowledgements}

The authors acknowledge valuable contributions by all members of CMI-HTA.

\section{References}

[1] S.T.C. Wong and H.K. Huang. A Hospital Integrated Framework for Multimodality Image Base Management. IEEE Trans. On Systems, Man, and Cybernetics, Vol. 26, No. 4, pp 455 -469, 1996.

[2] M. Tsiknakis, C.E. Chronaki, S. Kapidakis, C. Nikolaou, and S.C. Orphanoudakis, "An Integrated Architecture for the Provision of Health Telematic Services based on Digital Library Technologies", International Journal on Digital Libraries, Special Issue on "Digital Libraries in Medicine", vol. 1(3), 257-277, 1997.

[3] D.G. Katehakis, P. Lelis, E. Karabela, M. Tsiknakis, and S.C. Orphanoudakis: "An Environment for the Creation of an Integrated Electronic Health Record in HYGEIAnet, the Regional Health Telematics Network of Crete". TEPR 2000, Your Connection to Electronic Healthcare, San Francisco, May 2000.

[4] G. Potamias, M. Tsiknakis, D.G Katehakis, E. Karabela, V. Moustakis, and S.C. Orphanoudakis: Role-Based Access to Patients Clinical Data: The InterCare Approach in the Region of Crete. MIE Procs of MIE 2000 and GMDS 2000, Hanover, 1074-1079, Aug. 2000.

[5] C.E Chronaki, D.G. Katehakis, X. Zabulis, M. Tsiknakis, and S.C. Orphanoudakis: WebOnCOLL: An environment for collaboration in regional healthcare networks. IEEE Transactions on Information Technology in Biomedicine, 1(4), 257 269, 1997. 